\title{
Elaboração e análise sensorial de iogurte sabor sapoti (Manilkara zapota L.)
}

\author{
Suelma Regina dos Santos Luz ${ }^{1}$, Matheus Silva Alves², Mizael Calácio Araújo ${ }^{3}$, Marianna Basso Jorge4, \\ Naylanne Lima de Sousa ${ }^{5 *}$, Lívia Muritiba Pereira de Lima Coimbra ${ }^{6}$
}

\begin{abstract}
Resumo
A busca por alimentos saudáveis e nutritivos tem estimulado o setor de laticínios a desenvolver diferentes sabores de iogurtes, para atender as demandas de consumo da população. Adicionar polpa ou pedaços de frutas regionais é uma das estratégias utilizadas pela indústria alimentícia, com isso, o objetivo deste trabalho foi desenvolver iogurtes sabor sapoti e avaliar sua aceitabilidade e intenção de compra. Para tanto, foram desenvolvidas duas formulações de iogurte, com leite integral (F1) e leite desnatado (F2). As formulações, foram então, submetidas a análises microbiológicas para investigar presença de coliformes totais, fecais e microrganismos patogênicos, além de análise sensorial e intenção de compra, ambas através de Escala Hedônica. Foi calculado o Índice de Aceitabilidade para o atributo qualidade global. Na análise estatística os resultados foram submetidos ao teste " $t$ " de Student $(p<0,05)$ à nível máximo de significância de $5 \%$. As formulações apresentaram condições higiênico-sanitárias adequadas. Na avaliação sensorial foram consideradas bem aceitas ( $\geq 70 \%)$ no índice de aceitabilidade, sendo que as amostras se diferiram estatisticamente ( $\mathrm{p}<0,05)$ em todos os atributos avaliados (aroma, cor, sabor, textura e qualidade global), porém, a F1 obteve médias maiores em relação a F2. Desta forma, concluiu-se que as duas formulações de iogurte utilizando sapoti, obteviveram avaliações favoráveis por parte dos provadores, representando ótimas alternativas de consumo e aproveitamento do fruto.
\end{abstract}

Palavras-chave: Aceitabilidade. Produto Lácteo. Tecnologia de Alimentos. Fruta Tropical.

\section{Elaboration and sensory analysis of sapoti (Manilkara zapota L.) based yogurt}

\begin{abstract}
Healthy nutrition has stimulated the food industry to develop different yogurt flavors adding pulp or pieces of regional fruit to meet the consumption demands of the population. Thinking in the market strategies, the current work proposed to develop a tropical-flavored yogurt with sapoti (Manilkara zapota L.) fruit and evaluate its acceptability and purchase intention. Two yogurt formulations were developed, with whole milk (F1) and skimmed milk (F2). The samples were submitted to microbiological analysis to investigate the presence of total, fecal coliforms and pathogenic microorganisms, besides sensory analysis and purchase intention, both through of Hedonic Scale. The Acceptability Index was calculated for the global quality attribute and all the results were submitted to the Student's t-test ( $\mathrm{p}$ $<0.05$ ) at a maximum significance level of $5 \%$. As a result, both formulations presented adequate hygienic-sanitary conditions, sensorial evaluation accepted $(\geq 70 \%)$ by the acceptability index, and all others attribute evaluated (aroma, color, taste, texture, and overall quality) were significantly different between both formulations, with higher averages
\end{abstract}

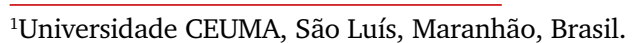

https://orcid.org/0000-0002-0626-941X

2PPG - BIONORTE - Rede de Biodiversidade e Biotecnologia da Amazônia Legal (UFMA) e Universidade CEUMA, São Luís, Maranhão, Brasil. https://orcid.org/0000-0002-4992-9588

${ }^{3}$ PPG - BIONORTE - Rede de Biodiversidade e Biotecnologia da Amazônia Legal (UFMA) e Universidade CEUMA, São Luís, Maranhão, Brasil. https://orcid.org/0000-0002-2720-8181

${ }^{4}$ PPG - BIONORTE - Rede de Biodiversidade e Biotecnologia da Amazônia Legal, Universidade Federal do Maranhão (UFMA), São Luís, Maranhão, Brasil.

https://orcid.org/0000-0002-8459-6707

${ }^{5}$ Universidade CEUMA, São Luís, Maranhão, Brasil.

https://orcid.org/0000-0002-0511-2330

${ }^{6}$ PPG - BIONORTE - Rede de Biodiversidade e Biotecnologia da Amazônia Legal (UFMA) e Universidade CEUMA, São Luís, Maranhão, Brasil. https://orcid.org/0000-0001-7926-417X

*Autor para correspondência: naylannelima@gmail.com

Recebido para publicação em 05 de novembro de 2019. Aceito para publicação em 27 de novembro de 2019. e-ISSN: 2447-6218 / ISSN: 2447-6218 / (C) 2009, Universidade Federal de Minas Gerais, Todos os direitos reservados. 
dos Santos Luz, S. R. et al.

to F1 compared to F2. That way, both formulations using sapoti fruit, obtained favorable results presenting as great alternatives of tropical yogurt flavor for the healthy nutrition market.

Keywords: Acceptability. Dairy Product. Food Technology. Topical Fruit.

\section{Introdução}

O Brasil é o quarto maior produtor de leite no mundo (Leite; Resende; Stock, 2019). A partir do leite, muitos derivados podem ser elaborados, tais como bebida láctea, queijo, doce de leite, manteiga, requeijão e iogurte, agregando valor ao produto (Farias et al., 2016). A busca dos consumidores por alimentos saudáveis e inovadores, tem proporcionado ao setor de laticínios, uma ampliação no desenvolvimento de novos produtos, dentre eles, o iogurte que contribui com o aumento da vida útil de prateleira, digestibilidade e manutenção do balanço gastrointestinal (Gonçalves et al., 2018).

O iogurte é uma excelente fonte de proteína, cálcio, fósforo, riboflavina, tiamina, vitamina B12, folato, niacina, magnésio e zinco (Das; Choudhary; Witrick, 2019) e tem sua formulação a partir da fermentação láctica, proveniente do cultivo das bactérias Streptococcus salivarius subsp. Thermophilus e Lactobacillus delbrueckii subsp. bulgaricus, conforme preconiza a legislação brasileira, podendo estar acompanhada de forma a complementar, outras bactérias ácido-lácticas, contribuindo nas características finais do produto (Brasil, 2007). O processo de adição de polpas de frutas no iogurte visa atenuar o sabor ácido e melhorar as características de aroma e textura, tornando-o mais nutritivo e atrativo ao consumo, encorajando o surgimento de novas pesquisas na área (Pádua et al., 2017).

Desta forma, tem-se adicionado polpas ou pedaços de frutas na produção de iogurtes, com a intenção de melhor aproveitar e contribuir com o aumento do consumo de frutas regionais, já existindo formulações adicionadas de polpa de abacaxi, acerola, morango e banana (Gonçalves et al., 2018; Munhoz et al., 2018). Dentre as frutas que também podem ser utilizadas, destaca-se o sapoti, opção ainda não explorada pela indústria alimentícia. O Sapotizeiro (Manilkara zapota L.) é originário de regiões quentes e úmidas da América Tropical, com potencial para exploração econômica no Brasil, com maior ênfase em pesquisas na região Nordeste, sendo o estado de Pernambuco o maior produtor nacional, outros estados como a Bahia, Pará, Ceará, Paraíba, Sergipe e Rio Grande do Norte também se destacam na produção do fruto (Melo et al., 2019; Alcântara et al., 2018; Silva Junior et al., 2014).

O sapoti é um fruto adocicado, rico em sacarose e frutose, vitaminas A, Complexo B, C e minerais cálcio, fósforo e ferro. Mas apesar de bastante nutritivo, o sapoti é muito perecível, sendo necessárias novas formas de processamento do fruto in natura, para que aumente sua estabilidade e permita sua comercialização (Coimbra et al., 2017; Bano e Ahmed, 2017). Dentro do panorama apresentado, visando agregar valor ao iogurte e obter mais uma alternativa de consumo e aproveitamento do fruto, o objetivo deste estudo foi elaborar iogurtes sabor sapoti, avaliando sua aceitabilidade e intenção de compra por parte dos consumidores.

\section{Material e métodos}

O estudo do tipo experimental analítico comparativo foi desenvolvido no Laboratório de Gastronomia, Laboratório de Microbiologia de Alimentos e Laboratório de Ciências do Ambiente - LACAM da Universidade CEUMA. Os frutos foram adquiridos em feira do Mercado Central localizado na região Central de São Luís - Maranhão, transportados para o Laboratório de Gastronomia onde foi dado prosseguimento nas etapas para elaboração do iogurte.

Inicialmente, os sapotis foram lavados em água corrente e sanitizados com solução de hipoclorito de sódio a 50 ppm, durante 10 minutos (Lemos et al., 2019). Logo após foi realizado enxágue em água corrente, os frutos foram cortados ao meio com uma faca de inox, para retirada das sementes e extração da polpa com auxílio de uma colher. Em seguida a polpa foi batida em liquidificador (Mallory) por 2 minutos, até formação de consistência levemente pastosa, colocada em sacos plásticos e armazenada no congelador (Consul). O processo de preparação da polpa é demonstrado na Figura 1.

Os demais ingredientes foram adquiridos no comércio local de São Luís - Maranhão, exceto o fermento lácteo liofilizado (Docina) que foi adquirido de fornecedor de Juiz de Fora - Minas Gerais. Sendo utilizado leite integral (Ultra High Temperature), leite desnatado (Ultra High Temperature), açúcar refinado, fermento lácteo (Docina) e polpa de sapoti.

Foram elaboradas duas formulações, uma contendo 3 litros de leite integral UHT (Ultra High Temperature), denominada como F1 (Formulação 1) e outra contendo 3 litros de leite desnatado UHT (Ultra High Temperature), denominada F2 (Formulação 2), acrescentando 8\% de açúcar refinado a cada formulação e submetida à fervura até alcançar a temperatura de $43^{\circ} \mathrm{C}$, aferida com auxílio de termômetro tipo espeto digital (Incoterm). Em seguida foi adicionado um sachê (3 gramas) de fermento lácteo liofilizado (Docina) para cada litro de leite utilizado nas formulações, colocadas em recipientes de vidro, e levadas para incubação em estufa (Quimis), à $43^{\circ} \mathrm{C}$, dando início ao processo de fermentação, por 10 horas, quando o $\mathrm{pH}$ de $4.7 \pm 1$ foi atingido. 
Elaboração e análise sensorial de iogurte sabor sapoti (Manilkara zapota L.)

Figura 1 - Processo de preparação da polpa de sapoti (Manilkara zapota L.)
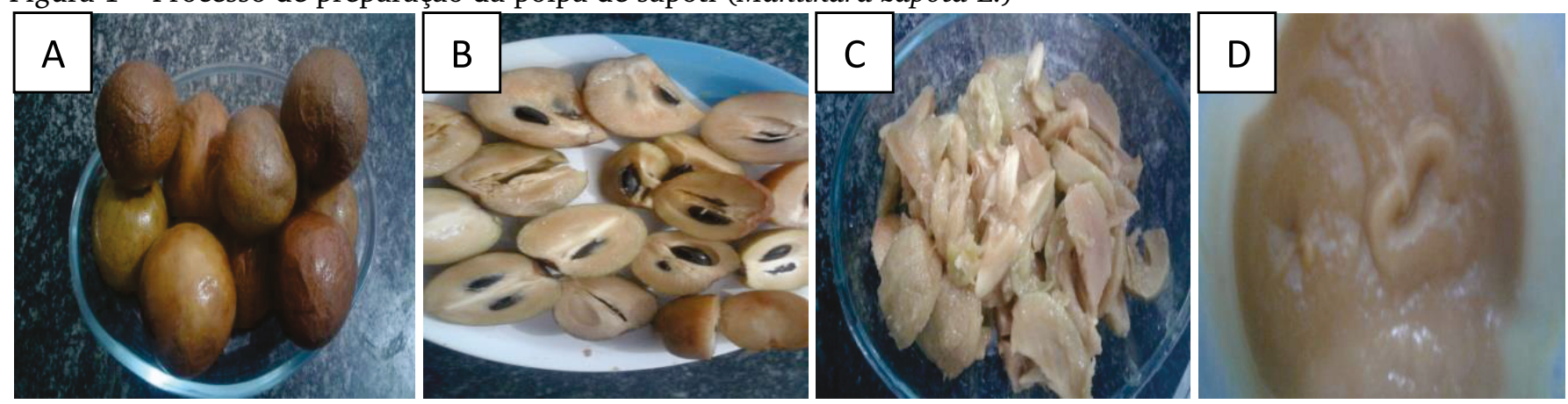

A: Frutos após sanitizados; B: Frutos cortados ao meio; C: Pedaços dos frutos sem casca e sementes;

D: Polpa após batimento. Fonte: Autoria própria (2019).

Após esse período, foram armazenadas sob refrigeração à $4^{\circ} \mathrm{C}$, em refrigerador (Consul), permanecendo por 16 horas para cessar o processo de fermentação e melhorar a consistência de iogurte. Logo se adicionou $25 \%$ de polpa de sapoti em cada formulação, sendo realizada manualmente a mistura da polpa com as formulações. Após a homogeneização, os iogurtes foram mantidos em refrigeração à $4^{\circ} \mathrm{C}$. Na Figura 2 , o processo de elaboração do iogurte pode ser visualizado de forma simplificada.

Figura 2 - Fluxograma de elaboração do iogurte sabor sapoti (Manilkara zapota L.)

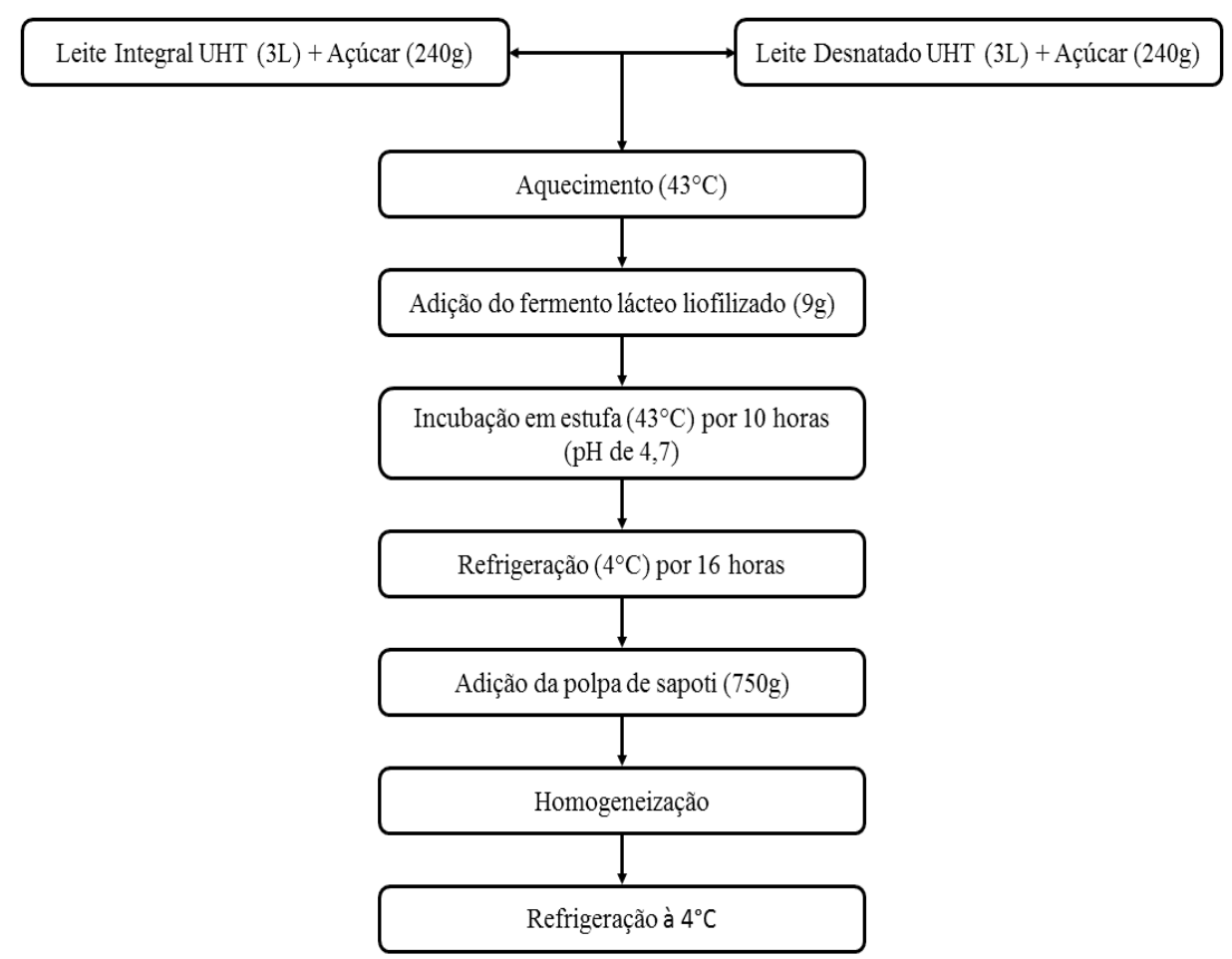

Com objetivo de verificar as condições higiênico-sanitárias, ou seja, a presença de microrganismos patogênicos e deteriorantes nos produtos elaborados, as duas formulações de iogurte foram submetidas à análise microbiológica através da Técnica de Tubos Múltiplos para determinação de coliformes a $30^{\circ} \mathrm{C}$, coliformes a $45^{\circ} \mathrm{C}$, contagem de fungos filamentosos e leveduras de acordo com a recomendação da Resolução $\mathrm{N}^{\circ} 12$, de 02 de janeiro de 2001 (Brasil, 2001).

Antes da análise sensorial, $50 \mathrm{~mL}$ das formulações de iogurte, foram transportadas em suas embalagens dentro de coller com bolsas de gelo e encaminhadas ao
Laboratório de Microbiologia de Alimentos da Universidade CEUMA. Inicialmente foram preparadas com $25 \mathrm{~g}$ diluições seriadas até $10^{-3}$ para uso na enumeração de fungos (fungos filamentosos e leveduras), coliformes totais e coliformes termotolerantes. A enumeração de fungos filamentosos e leveduras ocorreu pelo método de plaqueamento direto em superfície de cada diluição do alimento (até $10^{-3}$ ), em meio ágar batata dextrose, sendo os resultados expressos pelo número de Unidades Formadoras de Colônia por grama de amostra (UFC/g). Para a enumeração de coliformes totais, utilizou o teste presuntivo em caldo Lauril Sulfato Triptose (LST) e confirmatório em caldo Lactose Bile Verde Brilhante (VB), 
dos Santos Luz, S. R. et al.

com diluições seriadas (até $10^{-3}$ ) em triplicata, sendo ambos os caldos incubados em estufa a $35 \pm 2^{\circ} \mathrm{C}$ por 24 48 horas. De cada tubo positivo de LST, foi transferido um inóculo $(0,01 \mathrm{~mL})$ com o auxílio de alça para outro tubo contendo caldo EC, no qual foi incubado a $45 \pm 2^{\circ} \mathrm{C}$ por 24 horas (Gonçalves et al., 2018).

As formulações de iogurte foram submetidas a teste de aceitabilidade, com 70 provadores não treinados, de ambos os sexos, recrutados aleatoriamente entre acadêmicos e professores da Universidade CEUMA e com faixa etária entre 18 e 60 anos. Para realização da análise sensorial, a pesquisa foi inicialmente submetida ao Comitê de Ética em Pesquisa da Universidade CEUMA, sendo aprovada sob o número de processo 3.115.415. As análises aconteceram no Laboratório de Gastronomia da Universidade CEUMA, em cabines individualizadas. Os provadores receberam duas amostras codificadas por três dígitos, em copos plásticos contendo $50 \mathrm{ml}$ das formulações com temperatura em torno de $4^{\circ} \mathrm{C}$, água em temperatura ambiente e bolacha para limpeza do palato entre a prova das amostras.

O teste de aceitabilidade das amostras foi realizado através da Escala Hedônica de 9 pontos que variam de 1 "desgostei extremamente" a 9 "gostei extremamente". Com avaliação dos atributos: cor, sabor, aroma, textura e qualidade global (Instituto Adolfo Lutz, 2008). Para o teste de intenção de compra, utilizou-se a Escala Hedônica de 5 pontos que variam de 1 "jamais compraria" a 5 "certamente compraria" (Instituto Adolfo Lutz, 2008).
Foi calculado o índice de aceitabilidade para o atributo qualidade global (Dutcosky, 2013), utilizando a seguinte equação:

$$
I A(\%)=\frac{Y \times 100}{Z}
$$

Onde: $\mathrm{Y}=$ nota média obtida para o produto; $\mathrm{Z}=$ Nota máxima obtida.

Além das questões relacionadas à avaliação dos produtos elaborados, os provadores foram questionados sobre consumo e frequência de ingestão de iogurtes e benefícios de produtos lácteos para saúde. Os resultados obtidos para análise sensorial foram submetidos ao teste "t" de Student $(\mathrm{p}<0,05)$ a um nível de significância máximo de $5 \%$. Os demais resultados foram organizados com o auxílio do Programa Microsoft Excel® 2010 e em seguida foi realizado o desenvolvimento da análise estatística descritiva utilizando as variáveis categóricas descritas através de suas frequências absolutas (n) e relativas (\%).

\section{Resultados e discussão}

As análises microbiológicas indicaram que as duas formulações de iogurte, apresentaram condições higiênico-sanitárias satisfatórias, compatíveis aos critérios estabelecidos pela Resolução no 12 (Brasil, 2001) (tabela $1)$.

Tabela 1 - Análise microbiológica de iogurtes sabor sapoti (Manilkara zapota L.)

\begin{tabular}{|c|c|c|c|}
\hline Amostras & $\begin{array}{c}\text { Coliformes totais a } 30^{\circ} \mathrm{C} \\
(\mathrm{NMP} / \mathrm{g})\end{array}$ & $\begin{array}{l}\text { Coliformes fecais a } \\
45^{\circ} \mathrm{C}(\mathrm{NMP} / \mathrm{g})\end{array}$ & $\begin{array}{l}\text { Fungos filamentosos e } \\
\text { Leveduras (UFC/g) }\end{array}$ \\
\hline Legislação & - & 10 & - \\
\hline $\mathrm{F} 1$ & Ausente & Ausente & Ausente \\
\hline $\mathrm{F} 2$ & Ausente & Ausente & Ausente \\
\hline
\end{tabular}

NMP: número mais provável. UFC: unidades formadoras de colônias. F1 - formulação de iogurte com leite integral; F2 - formulação de iogurte com leite desnatado.

Para indicar que um alimento foi produzido em condições sanitárias adequadas é necessária a análise de bactérias mesófilas e coliformes, que são microrganismos presentes nas fezes e no ambiente, como solo, superfícies de vegetais, animais e utensílios. Tendo presença de coliformes a $45^{\circ} \mathrm{C}$, é um indicativo de condições higiênicas desfavoráveis e podem ocasionar as chamadas Doenças Transmitidas por Alimentos (DTAs), que são frequentemente associadas à ausência ou inadequada manipulação durante o processo de produção de alimentos (Oliveira et al., 2017; Gonçalves et al., 2018).

As médias das notas atribuídas pelos provadores não treinados para os diferentes atributos sensoriais estão expostos na Tabela 2. Houve diferença estatística $(\mathrm{p}<0,05)$ para todos os atributos avaliados (aroma, cor, sabor, textura e qualidade global), porém a formulação 1 apresentou médias maiores quando comparadas com a formulação 2 .

As formulações foram elaboradas de forma semelhante, utilizando a mesma quantidade de polpa de sapoti, mas com diferentes tipos de leite (integral e desnatado), ou seja, apresentaram diferentes concentrações de gordura. Como a Formulação 1 foi elaborada com leite integral, este fato pode ter influenciado na obtenção das maiores médias e maior aceitação por parte dos provadores.

Para Lopes et al. (2019) a aceitação de iogurtes é geralmente afetada pela sinérese (redução na formação do gel e aumento na separação do soro de leite), este 
Elaboração e análise sensorial de iogurte sabor sapoti (Manilkara zapota L.)

processo representa um dos principais defeitos visíveis na qualidade de iogurte. Os autores citam que aspectos como: taxa de acidificação, tipo de leite, teor de proteínas e tempo de fermentação podem influenciar as propriedades físicas e organolépticas do produto final. Já Ahmad et al. (2019) apontam que o efeito decrescente na sinérese pode ocorrer devido a maior quantidade de gordura presente no iogurte.

Tabela 2 - Média das notas do teste de aceitabilidade do iogurte sabor sapoti (Manilkara zapota L.)

\begin{tabular}{lccccc}
\hline Amostras & Aroma & Cor & Sabor & Textura & Qualidade Global \\
\hline F1 & $7,65^{\mathrm{b}}$ & $8,12^{\mathrm{b}}$ & $8,30^{\mathrm{b}}$ & $8,17^{\mathrm{b}}$ & $8,36^{\mathrm{b}}$ \\
F2 & $7,22^{\mathrm{a}}$ & $7,58^{\mathrm{a}}$ & $7,57^{\mathrm{a}}$ & $7,84^{\mathrm{a}}$ & $7,57^{\mathrm{a}}$ \\
\hline
\end{tabular}

Médias seguidas de letras iguais na vertical não diferem significativamente ao nível de 5\% de significância pelo teste "t" de Student. F1 - formulação de iogurte com leite integral; F2 - formulação de iogurte com leite desnatado.

No presente estudo, a formulação elaborada com leite integral (F1) foi melhor avaliada no atributo textura. Caracterizada por uma melhor consistência e viscosidade, podendo está estar associado ao teor de gordura da preparação. Resultados semelhantes foram encontrados por Sousa et al. (2019) ao analisar a aceitabilidade de iogurtes com leite integral e desnatado sabor açaí (Euterpe oleracea Mart.), sendo obtidas médias de 8,27 e 7,68 , respectivamente.

$\mathrm{O}$ atributo aroma apresentou médias menores em relação aos demais atributos. Bessa e Silva (2018) afirmam que o aroma é um atributo importante para produtos formulados com frutas, já que o consumidor $\mathrm{o}$ associa diretamente à fruta que foi utilizada, sendo um fator decisivo na sua escolha. Durante o processo de avaliação do estudo em questão, alguns provadores relataram que o iogurte remetia ao cheiro de ameixa, o que pode ter contribuído para menores valores nas médias de ambas as formulações.

Já a cor é uma das qualidades que mais se destacam, sendo considerada a apresentação visual do alimento e representa o primeiro contato do consumidor e o produto, este atributo é capaz de despertar determinadas reações e sensações pessoais de aceitação, indiferença ou rejeição, refletindo diretamente na escolha e aceitação do produto (Barboza e Cazal, 2018). A polpa do sapoti conferiu uma cor que agradou os provadores na avaliação. $\mathrm{O}$ atributo cor obteve médias variando entre 7,58 para formulação com leite desnatado e 8,12 para formulação com leite integral, sendo classificadas na faixa de aceitação da escala hedônica como "gostei regularmente" e "gostei moderadamente", respectivamente.

Gonçalves et al. (2017) compreendem por paladar quando os receptores da boca, localizados principalmente na língua, são expostos às substâncias químicas encontradas na composição de alimentos e produzem sensações de sabor. Já Santos, Cruz e Almeida (2017) citam que a textura, aparência e sabor, são as características sensoriais que mais se destacam na aquisição e consumo de produtos alimentícios, contribuindo também para o monitoramento da qualidade final do produto. Quando avaliado o atributo sabor, a formulação 1 obteve média maior $(8,30)$ em relação a formulação $2(7,57)$.
Para que um produto seja considerado aceito, em termos de suas propriedades sensoriais, é necessário que se obtenha um índice de aceitabilidade de no mínimo 70\% (Dutcosky, 2013). Quando calculado o índice de aceitabilidade para o atributo qualidade global, foram obtidos $92,8 \%$ (F1) e $84,1 \%$ (F2), valores estes $\geq 70 \%$, considerados assim produtos aceitos sensorialmente (Figura 3). Sousa et al. (2019), ao avaliarem a qualidade global de iogurtes sabor açaí (Euterpe oleracea Mart.) também obtiveram resultados favoráveis para ambas formulações, apresentando $87 \%$ para formulação com leite desnatado e $91 \%$ para formulação com leite integral.

Ao serem questionados sobre o consumo de iogurtes, $90 \%$ dos avaliados relataram que consomem iogurtes, $41 \%$ afirmaram consumir pelo menos uma vez por semana, $57 \%$ preferem sabor morango, apenas $2,85 \%$ acreditam que iogurte não faz bem à saúde e $74 \%$ relataram conhecer os benefícios de produtos lácteos para saúde. Os benefícios mais citados foram: fonte de cálcio, fortalecimento dos ossos e melhoria do trânsito intestinal, auxiliando na digestão. Os resultados demonstram que a maioria dos entrevistados conhecem os benefícios de produtos lácteos para saúde e o consomem regularmente, mesmo que ainda tenham preferência pelo sabor mais tradicional.

Em relação à pesquisa de mercado realizada, descrita como intenção de compra, observou-se resultados favoráveis para as duas formulações, sendo que a F1 obteve maior percentual de "certamente compraria" (81\%) e não apresentou classificação desfavorável, "jamais compraria" (Tabela 3). Desta forma, pode-se afirmar que as formulações de iogurtes avaliadas apresentaram bom potencial de consumo, sendo que a Formulação 1 obteve média mais elevada em relação à Formulação 2, já que a maior porcentagem de provadores atribuiu uma intenção de compra como "Certamente compraria". 
Figura 3 - Índice de aceitabilidade para o atributo qualidade global do iogurte sabor sapoti (Manilkara zapota L.)

\section{TESTE DE ACEITABILIDADE}

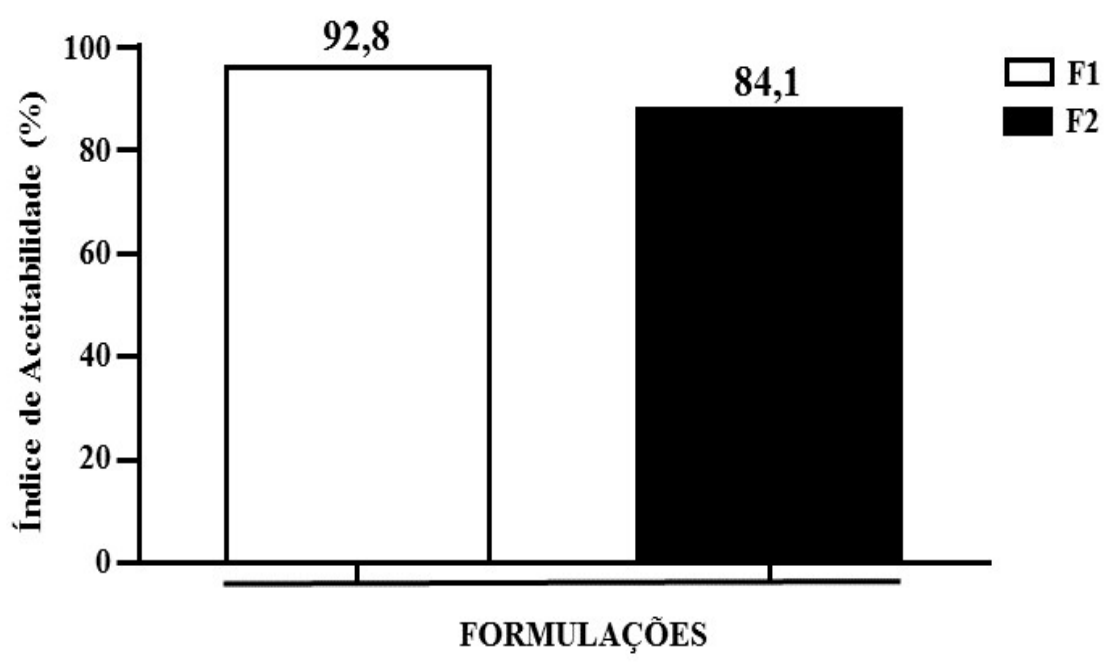

F1 - formulação de iogurte com leite integral; F2 - formulações de iogurte com leite desnatado.

Tabela 3 - Percentual das notas obtidas na intenção de compra para a Formulação 1 e Formulação 2 de iogurte sabor sapoti (Manilkara zapota L.)

\begin{tabular}{cccccc}
\hline & \multicolumn{2}{c}{ Percentual (\%) } & & \\
\hline Amostras & $\begin{array}{c}\text { Jamais } \\
\text { compraria }\end{array}$ & $\begin{array}{c}\text { Talvez não } \\
\text { compraria }\end{array}$ & $\begin{array}{c}\text { Talvez compraria/ } \\
\text { Talvez não compraria }\end{array}$ & $\begin{array}{c}\text { Talvez } \\
\text { compraria }\end{array}$ & $\begin{array}{c}\text { Certamente } \\
\text { compraria }\end{array}$ \\
\hline F1 & 0 & 1 & 9 & 9 & 81 \\
\hline F2 & 3 & 10 & 10 & 44 & 33 \\
\hline
\end{tabular}

F1 - formulação de iogurte com leite integral; F2 - formulação de iogurte com leite desnatado.

\section{Conclusão}

As duas formulações de iogurtes apresentaram condições higiênico-sanitárias satisfatórias e obtiveram resultados favoráveis na avaliação de aceitabilidade e intenção de compra. Sendo que, a formulação com leite integral foi melhor avaliada e aceita pelos provadores, em relação à formulação com leite desnatado. Portanto, a nova formulação de iogurte sabor sapoti surge como mais uma alternativa de aproveitamento do fruto, que beneficiará a população, através da elaboração de um produto com gosto regional, além de contribuir para surgimento de novas pesquisas e motivar o aumento no consumo de sapoti e iogurte.

\section{Aprovação do Comitê de Ética}

O projeto foi aprovado pelo Comitê de Ética em Pesquisa da Universidade CEUMA, sob protocolo no 3.115.415.

\section{Referências}

Ahmad, I.; Xiong, Z.; Xiong, H.; Din, Z. U.; Javaid, A. B.; Nawaz, A.; Walayat, N. 2019. Enzymatic Hydrolyzed Potato Powder Stabilizes the Properties and Rheological Performance of Yogurt. Preprints, 2019080005. Doi: https://doi.org/10.20944/preprints201908.0005.v1.

Alcântara, D. B.; Paz, M. S. O.; Rodrigues, T. H. S.; Fernandes, T. S. M.; Barbosa, P. G. A.; Loiola, A. R.; Grinberg, P.; Zocolo, G. J.; Britof, E. S.; Nascimento, R. F. 2018. Organophosphorus Pesticide in Sapodilla (Manilkara zapota) Fruit. Journal of the Brazilian Chemical Society, 29: 2180-2188. Doi: http://dx.doi.org/10.21577/0103-5053.20180094.
Bano, M.; Ahmed, B. 2017. Manilkara zapota (L.) P.Royen (Sapodilla): A Review. International Journal of Advance Research, Ideas and Innovations in Technology, 3: 1364-1371. Disponível em: https://www. ijariit.com/manuscript/manilkara-zapota-l-p-royen-sapodilla-a-review/.

Barboza, H. C.; Cazal, M. M. 2018. Avaliação da influência de características sensoriais e do conhecimento nutricional na aceitação do chá-mate. Brazilian Journal of Food Technology, 21: e2017075. Doi: http://dx.doi.org/10.1590/1981-6723.7517. 
Elaboração e análise sensorial de iogurte sabor sapoti (Manilkara zapota L.)

Bessa, M. M.; silva, A. G. F. 2018. Elaboração e caracterização físicoquímico e sensorial de iogurte prebiótico de tamarindo. Revista do Instituto de Laticínios Cândido Tostes, 73: 185-195. Doi: http://dx.doi. org/10.14295/2238-6416.v73i4.581.

Brasil. Ministério da Agricultura, Pecuária e Abastecimento. Instrução Normativa no 46, de 23 de outubro de 2007 [MAPA]. Regulamento Técnico de Identidade e Qualidade de Leites Fermentados. DOU n ${ }^{\circ} 205$, Seção 1, p.4. Brasília, DF, 2007. Disponível em: https://www. jusbrasil.com.br/diarios/741136/pg-4-secao-1-diario-oficial-da-uniaodou-de-24-10-2007.

Brasil. Ministério da Saúde. Agência Nacional de Vigilância Sanitária. Resolução № 12, de 02 de janeiro de 2001 [ANVISA]. Regulamento técnico sobre padrões microbiológicos para alimentos. Rio de Janeiro, 2001. Disponível em: http://portal.anvisa.gov.br/documents/33880/2568070/ RDC_12_2001.pdf/15ffddf6-3767-4527-bfac-740a0400829b.

Coimbra, L. M. P. L.; Arruda, H. A. S.; Machado, E. C. L.; Salgado, S. M.; Albuquerque, S. S. M. C.; Andrade, S. A. C. 2017. Water and sucrose diffusion coefficients during osmotic de hydration of sapodilla (Achras zapota L.). Revista Ciência Rural, 47: 1-7. Doi: http://dx.doi. org/10.1590/0103-8478cr20150924.

Das, K.; Choudhary, R.; Witrick, K. A. T. 2019. Effects of new technology on the current manufacturing process of yogurt-to increase the overall marketability of yogurt. LWT - Food Science and Technology, 108: 69-80. Disponível em: https://doi.org/10.1016/j.lwt.2019.03.058.

Dutcosky, S. D. 2013. Análise Sensorial de Alimentos. 4. ed. Champagnat, Curitiba.

Farias, P. K. S.; Nogueira, G. A. B.; Santos, S. G. A.; Prates, R. P.; Silva, J. C. R.; Souza, C. N. 2016. Contagem de bactérias lácticas em iogurtes comerciais. Caderno de Ciências Agrárias, 8: 38-44. Disponível em: https://periodicos.ufmg.br/index.php/ccaufmg/article/ view/2939/1777.

Gonçalves, A. O. S.; David, G. Q.; Silva, M. P.; Peres, W. M.; Yamashita, O. M. 2017. Avaliação sensorial e aceitação comercial do cogumelo comestível Pleurotus florida. Revista de Agricultura Neotropical, 4: 92-98. Disponível em: https://periodicosonline.uems.br/index.php/ agrineo/article/view/1614/1780.

Gonçalves, N. M.; Ferreira, I. M.; Silva, A. M. O.; Carvalho, M. G. 2018. Iogurte com geleia de cajá (Spondias mombin L.) adicionado de probióticos: avaliação microbiológica e aceitação sensorial. Revista Brasileira de Higiene e Sanidade Animal, 12: 169-178. Disponível em: http://www.higieneanimal.ufc.br/seer/index.php/higieneanimal/ article/view/440.

Instituto Adolfo Lutz. 2008. Métodos físico-químicos para análise de alimentos. 4. ed. IAL, São Paulo. Disponível em: http://www.ial.sp.gov. br/resources/editorinplace/ial/2016_3_19/analisedealimentosial_2008. pdf.
Silva Junior, J. F. S.; Bezerra, J. E. F; Lederman, I. E.; Moura, R. J. M. 2014. O sapotizeiro no Brasil. Revista Brasileira de Fruticultura, 36: p.86-99. Doi: http://dx.doi.org/10.1590/0100-2945-449/13.

Leite, J. L. B.; Resende, J. C.; Stock, L. A. 2019. Desempenho do mercado brasileiro de lácteos. Anuário Leite 2019. São Paulo. Disponível em: http://ainfo.cnptia.embrapa.br/digital/bitstream/item/198698/1/ Anuario-LEITE-2019.pdf.

Lemos, D. M.; Rocha, A. P. T.; Gouveia, J. P. G.; Oliveira, E. N. A.; Sousa, E. P.; Silva, S. F. 2019. Elaboração e caracterização de geleia prebiótica mista de jabuticaba e acerola, Brazilian Journal of Food Technology, 22: e2018098. Doi: http://dx.doi.org/10.1590/1981-6723.09818.

Lopes, R. P.; Mota, M. J.; Pinto, C. A.; Sousa, S.; Silva, J. A. L.; Gomes, A. M.; Delgadillo, I.; Saraiva, J. A. 2019. Physicochemical and microbial changes in yogurts produced under different pressure and temperature conditions. LWT - Food Science and Technology, 99: 423-430 Doi: https://doi.org/10.1016/j.lwt.2018.09.074.

Melo, L. D. F. A.; Melo Junior, J. L. A.; Ramos, M. G. C.; Assis, W. O.; Paes, R. A.; Andrade, A. P; Soares, L. B. F.; Costa, J. F. O.; Souto. P. C.; Neves, M. I. R. S.; Rocha, D. F. 2019. Biometry and Pre-germinating Manilkara zapota L. Seed Treatments. Journal of Agriculture and Ecology Research International, 19: 1-9. Doi: https://doi.org/10.9734/ jaeri/2019/v19i430091.

Munhoz, C. L.; Borges, G. S.; Silva, M. L. F; Oliveira, R. F. 2018. Avaliação sensorial de iogurtes de jambo vermelho. Revista Inova Ciência \& Tecnologia, 4: 25-31. Disponível em: http://periodicos.iftm.edu.br/ index.php/inova/article/view/446/256.

Oliveira, J. F.; Garcia, L. N. H.; Pastore, V. A. A.; Raghiante, F; Possebon, F. S.; Pinto, J. P. A. N.; Martins, O. A. 2017. Qualidade de iogurtes de coco e morango. Revista Brasileira de Higiene e Sanidade Animal, 11: 416-425. Disponível em: http://www.higieneanimal.ufc.br/seer/index. $\mathrm{php} /$ higieneanimal/article/view/416.

Pádua, H. C.; Silva, M. A. P.; Souza, D. G.; Moura, L. C.; Plácido, G. R.; Couto, G. V. L.; Caliari, M. 2017. Iogurte sabor banana (Musa $\mathrm{AAB}$, subgrupo prata) enriquecido com farinha da casca de jabuticaba (Myrciaria jabuticaba (Vell.) Berg.). Global Science and Technology, 10: 89-104. Disponível em: https://rv.ifgoiano.edu.br/periodicos/index. $\mathrm{php} / \mathrm{gst} / \mathrm{article} / \mathrm{view} / 861$.

Santos, M. V.; Cruz, R. G.; Almeida, M. E. F. 2017. Desenvolvimento e avaliação sensorial de iogurte com sementes de chia. Revista do Instituto de Laticínios Candido Tostes, 72: 01-10. Doi: https://dx.doi. org/10.14295/2238-6416.v72i1.535.

Sousa, N. L.; Alves, M. S.; Gomes, W. C.; Zagmignan, A.; Silva, L. C. N.; Ferreira, L. C.; Santos, A. F.; Coimbra, L. M. P. L. 2019. Elaboração e análise sensorial de iogurte sabor açaí (Euterpe oleracea Mart.). p. 260-268. In: Silva Neto, B. R. S., A Produção do Conhecimento nas Ciências da Saúde 2, Atena Editora, Ponta Grossa. Disponível em: https://www.atenaeditora.com.br/arquivos/ebooks/a-producao-doconhecimento-nas-ciencias-da-saude- 2 . 